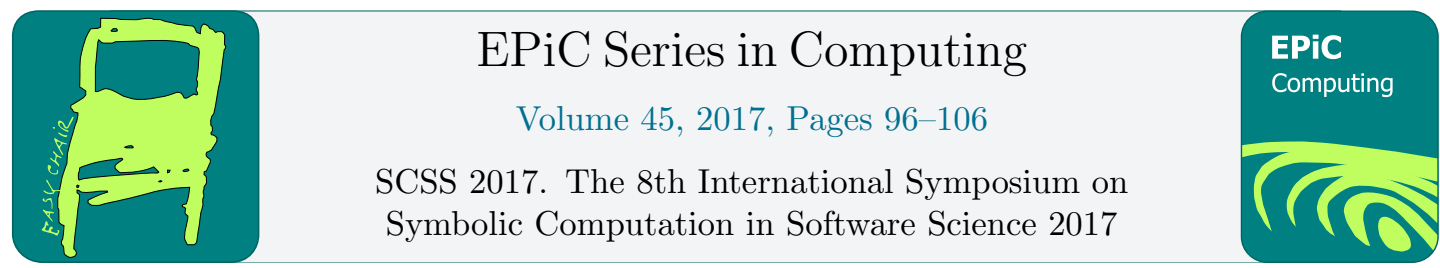

\title{
Formal Development of Distributed Enumeration Algorithms By Refinement-Based Techniques
}

\author{
Maha Boussabbeh ${ }^{1,2}$, Mohamed Tounsi², \\ Mohamed Mosbah ${ }^{1}$, and Ahmed Hadj Kacem² \\ 1 LaBRI Laboratory, CNRS, Bordeaux INP, University of Bordeaux, France \\ $\{$ maha. bousabbah, mohamed.mosbah\}@u-bordeaux.fr \\ 2 ReDCAD Laboratory, University of Sfax, Tunisia \\ \{mohamed.tounsi, ahmed.hadjkacem\}@fsegs.rnu.tn
}

\begin{abstract}
The enumeration problem addresses a collection of important algorithmic issues related to distributed computations. Among existing solutions, we are interested on the seminal algorithm of Mazurkiewicz, based on local computations. Our paper contributes to the design of a correct-by-construction enumeration algorithm. The main idea relies upon the development of the problem following a top/down approach that can be supported by an incremental process controlled by the refinement of models. Event-B modelling language is supporting our methodology. Our main objective is to provide a verified component for distributed enumeration in order to be used and extended for solving other problems of distributed algorithms.
\end{abstract}

\section{Introduction}

\subsection{Overview}

Distributed algorithms [14] are designed to run on interconnected autonomous computing entities for achieving a common task: each entity executes asynchronously the same code and interacts locally with its immediate neighbours. Local computations [10], [4], [6], based on graph rewriting, facilitate the design of these algorithms. A distributed system is modelled with respect to the local computations model, by a simple, connected and undirected graph where nodes denote processors and edges denote communication links. Local computations provide an abstract model to reason about problems independently of the network topology.

The naming problem is a fundamental aspect in distributed computing, since it produces a final configuration where all entities have unique identities. Such a configuration is motivated by several computations and applications which require the assumption that all entities should be unambiguously identified. Therefore it constitutes basic initial steps of many other distributed algorithms. Enumeration problem is a variant of the naming problem. It aims to give each entity a unique number between 1 and the size of the graph.

Several solutions for the enumeration problem have been published, among them we consider the seminal algorithm of Mazurkiewicz [11]. Mazurkiewicz's algorithm, based on local 
computations, enumerates nodes in an anonymous minimal-covering graph. It is applied on the asynchronous computation model where nodes exchange their states (labels) and accordingly to those, they do a computation step and update local states. In one computation step, labels of nodes are modified on a sub-graph consisting of a node and its neighbours, according to rules depending on this sub-graph only.

\subsection{Related Work}

Mazurkiewicz's algorithm has been studied to give pertinent solutions to a large family of problems. Among these problems, the distributed election constitutes a building block of many other algorithms. It is clearly related to the enumeration problem. A distributed algorithm solves the election problem if in the final configuration, exactly one node is marked as elected and all other nodes are marked as non-elected. Given an enumeration, we can obtain an election algorithm by considering the node with the highest/lowest identifier as elected. Based on the enumeration algorithm, authors in [7] introduced the notion of quasi-covering, i.e., which captures the existence of large enough area of one graph that looks locally like another graph, and characterised families of graphs which admit an election algorithm in the message passing model. Authors in [9] presented a new algorithm, based on Mazurkiewicz's one for the selfstabilizing enumeration. Enumeration problem has been also studied for snapshot problems and detection of stable properties in anonymous networks. Most known snapshot algorithms assume that vertices of network have unique identifier and/or there is exactly one initiator. Based on the enumeration process, authors in [8] explained what stable properties of a distributed system can be computed anonymously with local snapshots with multiple initiators.

Formal methods provide a real help for expressing correctness with respect to safety properties in the design of distributed computing. Nevertheless, specifications of a complex system, become very large and difficult to understand. The correct-by-construction [12] approach provides an incremental proof-based process to construct and prove algorithms. Its main idea relies upon a formal development following a top/down approach. This technique can be supported by a progressive and incremental process controlled by the refinement of models. A growing activity on correct-by-construction developments has started and is addressing many kinds of case studies and problems in different fields: formalizing snapshot algorithms [3], investigating formal patterns for proof-based development [15], analysing protocols [13], etc.

\subsection{Contribution}

In this paper, we are interested on the study of Mazurkiewicz's algorithm. We explore the correct-by-construction process to formalize enumeration problem in distributed systems. The main objective is to solve the problem using refinement techniques and provide a solution which can be reused for conceiving correct algorithms based on a correct enumeration. We start with an abstract initial specification of the problem and we enrich it gradually by a progressive and incremental refinement. Several refinement steps allow to capture the complete and desired behaviour of the algorithm. The refinement of models is the key element allowing preservation of properties between the different levels of abstraction. The Event-B [1] modelling language is supporting this methodology proposal suggesting proof-based guidelines using the RODIN plateform [2]. 


\subsection{Organization Of The Paper}

The paper is organized as follows: Section 2 recalls basic concepts of the local computation models and Event-B method. Section 3 gives a description of Mazurkiewicz's algorithm. Section 4 introduces the refinement process of the algorithm where we describe different abstraction levels. Section 5 details formal specifications of each level. Finally, a short conclusion and ongoing work round the paper up.

\section{Preliminaries}

\subsection{Local Computations Model}

In this section, we illustrate, in an intuitive way, the notions of local computations, and particularly those of graph relabelling systems by showing how some algorithms on networks of processors may be encoded within this framework. As usual, such a network is represented by a graph whose vertices stand for processors and edges for (bidirectional) links between processors. At every time, each vertex and each edge is in some particular state and this state will be encoded by a vertex or an edge label. According to its own state and to the states of its neighbours, each vertex may decide to realize an elementary computation step. After this step, the states of this vertex, its neighbours and the corresponding edges may be changed according to some specific computation rules. The graph relabelling systems meet the following requirements:

- they do not change the underlying graph but only the labelling of their components (edges and/or vertices), the final labelling being the result,

- they are local, that is, each rewriting changes only a connected subgraph of a fixed size in the underlying graph. Given $G$ a graph, a subgrph contains a subset of the vertices and edges in $G$,

- they are locally generated, that is, the applicability condition of the rewriting depends only on the local context of the relabelled subgraph.

\subsection{The Modelling Framework}

The Event-B [1] modelling language defines mathematical structures as contexts and formal model of the system as machines. The context is defined by abstract sets, constants, and axioms which describe constant properties. An Event-B machine describes a reactive system by a set of invariant properties and a finite list of events modifying state variables. An invariant is defined as a predicate that holds in all reachable states. An event is decomposed into a guard that specifies under which circumstances it might occur and some generalized substitutions called actions that define the state transition associated with the event. A machine $M$ may see a context $C$, this means that all carrier sets and constants defined in $C$ can be used in $M$. A context $C^{\prime}$ can extend a context $C$, this means that all properties defined in $C^{\prime}$ are added to C.

Event-B uses a top-down refinement-based approach. The refinement [5] of a specification allows to enrich it in a step-by-step fashion. It provides a way to strengthen invariants and add details to a model. It is used to transform an abstract model into a more concrete version by modifying the state description. This is done by extending the list of state variables, by refining 
each abstract event into a corresponding concrete version, and by adding new events. In Fig. 1 , the diagram illustrates the refinement-based relationship among events and models.

We suppose that an abstract model $A M$ with variables $x$ and invariant $I(x)$ is refined by a concrete model $C M$ with variables $y$. The abstract state variables, $x$, and the concrete ones, $y$, are linked together by means of the so-called gluing invariant $J(x, y)$. A number of proof obligations ensure that (1) each abstract event of $A M$ is correctly refined by its corresponding concrete version of $C M$, (2) each new event of $C M$ refines skip, which is intending to model hidden actions over variables appearing in the refinement model $C M$. More formally, if $B A_{-} a e\left(x, x^{\prime}\right)$ and $B A_{-} c e\left(y, y^{\prime}\right)$ are respectively the abstract and concrete before-after predicates of events, we say that $c e$ in $C M$ refines $a e$ in $A M$ or that $c e$ simulates $a e$, if one proves the following statement corresponding to proof obligation: $I(x) \wedge J(x, y) \wedge B A_{-} c e\left(y, y^{\prime}\right) \Rightarrow$ $\exists x^{\prime} \cdot\left(B A_{\_} a e\left(x, x^{\prime}\right) \wedge J\left(x^{\prime}, y^{\prime}\right)\right)$. To summarize, refinement guarantees that the set of traces of the abstract model $A M$ contains (modulo stuttering) the traces of the concrete model $C M$.

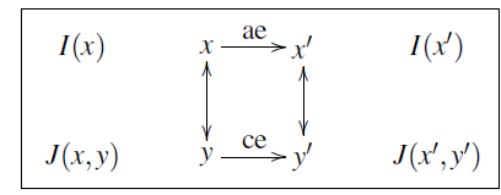

Figure 1: Refinement-Based Relationship

\section{A Distributed Enumeration Algorithm}

Distributed enumeration algorithms are considered to solve distributed naming problems. The aim of such algorithms is to attain a final configuration where all processes have unique identities. In this section we present a description of an enumeration algorithm given by Mazurkiewicz [11].

Given $G$ an undirected graph, i.e., no distinction between two nodes associated with an edge; $N(G)$ stands for the nodes of $G$ with a cardinality denoted by $|N(G)|$. During the execution of the algorithm, each node $x$ attempts to get its own unique identity which is a number between 1 and $|N(G)|$. Initially every node is defined by a 0 -identity: it does not get a number yet. Once a node $x$ has chosen a number $n(x)$, it sends it to each neighbour $y$. When $y$ receives a message from $x$, it stores the number $n(x)$. A Local view can be constructed by each node $x$ of the graph, containing all numbers of its neighbours. The set of Local views of neighbours numbers stands for the MailBox of $x$. Each node broadcasts its number and its MailBox. If a node $x$ discovers the existence of another node $y$ with the same number (identity), then it should decide if it changes its identity: it compares its Local view with the Local view of $y$. If the Local view of $y$ is weaker, then $y$ picks another number and broadcasts it with its Local view. At the end of the execution, we can construct a graph $H$ whose vertices are defined by all $G$ node identities. If $G$ is a minimal covering of $H$, then the algorithm terminates correctly and every node has a unique number.

A total order should be defined on Local views of nodes: let $N 1$ and $N 2$ be respectively the set of Local view of $x$ and $y$. Let $E$ be the set of elements of the symmetric difference of $N 1$ and $N 2$. $E=(N 1 \backslash N 2) \cup(N 2 \backslash N 1)$. Formally, $N 1$ is weaker than $N 2$ if the maximum of elements of $E$ belongs to $N 2$.

During the execution, the label of each node $x$ is a tuple $(n(x), L v(x), M b(x))$ where: $n(x)$ is the current number of $x$ computed by the algorithm; $L v(x)$ is the Local view of $x$. It is a set of 
identities of nodes connected to $x . M b(x)$ is the MailBox of $x$. It is a set of neighbour identities and their Local views.

Based on local computations [10], Mazurkiewicz [11] defines some computation rules applied on nodes in order to change their numbers, when needed, and diffuse their own information. Given $x$ a node of the graph, the ball around $x$, denoted by $B(x)$ is the set of nodes covering $x$ and its neighbours. Mazurkiewicz distinguishes two rules applied on $B(x)$ as follows:

The Renaming Rule describes the instruction that a node has to follow when the Mailbox of elements of $B(x)$ is not modified; and it has not a number yet, or when it discovers the existence of another Local view $N^{\prime}$ greater than its own. In such cases, this node chooses another number, updates its Local view by removing elements which corresponds to its old number and adding the chosen one. Then, every node of $B(x)$ should update their Mailbox.

Renaming Rule:

Pre-conditions:

$\forall y \in B(x), M b(y)=M b(x)$

$(n(x)=0) \vee\left(n(x)>0 \wedge \exists\left(n(x), N^{\prime}\right) \in M(x) \mid N(x)<N^{\prime}\right)$

Post-conditions:

$n^{\prime}(x)=1+\max \{n \mid(n, N) \in M(x)\}$

$\forall y \in B(x) \backslash\{x\}, N^{\prime}(y)=(N(y) \backslash\{n(x)\}) \cup\left\{n^{\prime}(x)\right\}$

$\left.\forall y \in B(x), M^{\prime}(y)=M(y) \cup\left\{n^{\prime}(w), N^{\prime}(w)\right) \mid w \in B(x)\right\}$

If the Mailbox has been modified, then nodes of $B(x)$ should diffuse their informations and update their Mailbox.

Diffusion Rule:

Pre-conditions:

$\exists y \in B(x), M b(y) \neq M b(x)$

Post-conditions:

$\forall y \in B(x), M^{\prime}(y)=\bigcup_{w \in B(x)} M(w)$

\section{Incremental Development}

In this section we present the complete refinement process (Figure. 2) which starts from GLOBAL-ENUM and NETWORK and progressively leads to LOCAL-ENUM-PROCESS. NETWORK is an event-B context specifying the application field of the enumeration algorithm. The network is represented by a graph whose vertices stand for processors and edges for (bidirectional) links between processors. The network is supposed to be fixed (edges and nodes are not modified or created or deleted). Moreover, it should be noted that Mazurkiewicz's algorithm terminates correctly on graphs that are simple, connected, undirected and minimal for the covering relations. The GLOBAL-ENUM model is an abstract event-B machine specifying the result of the algorithm in one shot (Figure. 3) without specifying the algorithmic process: each node picks a unique identity. The GLOBAL-PROGRESS-ENUM model is an event-B machine refining GLOBAL-ENUM. It introduces in a high level abstraction the basic idea for computing the result in a progressive way. In this level, we take a global view on the graph and we fix a number called maxId. This number is initialized to 0 and reaches gradually the cardinality of the graph $|N(G)|$. The progressive aspect comes from the fact that all numbers that are less than maxId should be allocated. By this way, we ensure the surjective property of the enumeration. The LOCAL-PROGRESS-ENUM model is an event-B machine refining the previous level. It takes a local view on the graph and explains how the the progressive computation is working on balls of the graph. This level ensures the injective property of the enumeration restricted to the balls of the graph. The LOCAL-RENAM-PROCESS model is an event-B machine refining 


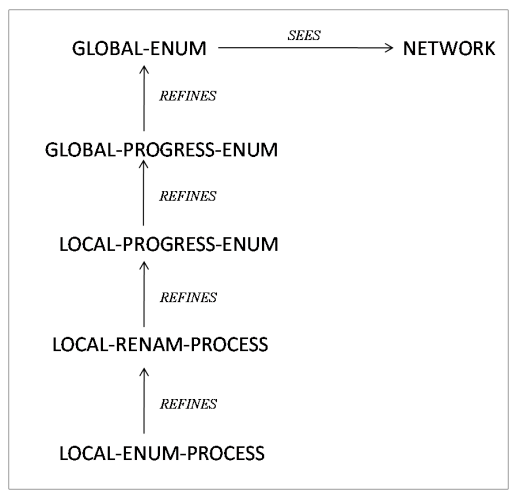

Figure 2: A Refinement Process

the previous one. It introduces an abstract definition of the Renaming rule and explains how nodes can construct their Local views from their neighbours. The LOCAL-ENUM-PROCESS model refines the LOCAL-RENAM-PROCESS and ensures the correctness of the algorithm. It computes through an event-B machine a concrete definition of the Renaming and the Diffusion rules of the algorithm. MailBox of nodes are constructed in this level.

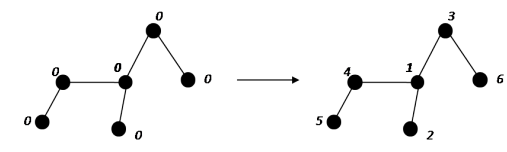

Figure 3: Enumeration of nodes in one shot

\section{Formal Development}

\subsection{Network Specification}

In this section we introduce formal specifications of a network on which the distributed enumeration algorithm can be executed. An undirected graph means that there is no distinction between two nodes associated with an edge (see axm4). A graph $g$ is simple, if it has at most one edge between any two nodes (see axm2 and axm3) and no edge starts and ends at the same node (see axm5); it is obviously expressed by the choice of the relation representation by a set. The domain restriction $N D \triangleleft i d$ is a subset of the relation $i d$ that contains all of the pairs whose first element is in $N D ; i d$ is the identity relation that maps every element to itself. A graph (directed or not) is connected, if for each pair of nodes, there exists a path joining these two nodes (see axm6). A connected graph $g$ over a set of finite nodes $N D$ (see $a x m 1$ ) can be presented as follows:

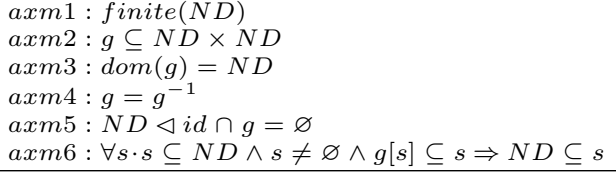

It has been proved that enumeration algorithms cannot be running on ambiguous graphs [11]. The notion of ambiguity is formulated equivalently using coverings of simple graphs. 
Mazurkiewicz's algorithm terminates correctly on graphs that are minimal for the covering relations: they can cover only themselves. Formally, a graph $g$ is a covering of another graph $H$, if there is a surjective homomorphism phi from $g$ to $H$ (axm7: line 2$)$ which is locally bijective (axm7: lines 3, 4 and 5). $g$ is minimal if phi is an isomorphism: phi is a bijection (axm7: line $6)$. Therefore, we add the following axiom to ensure that if $g$ is a covering of $H$, then $g$ and $H$ are isomorphic via the homomorphism phi

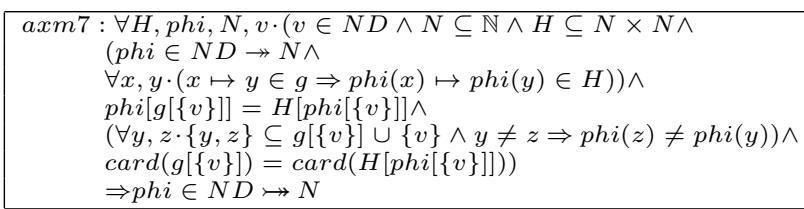

\subsection{The Initial Model}

The first level of the model expresses only the goal of the distributed algorithm and does not describe how the solution is computed. This level can be defined by an abstract machine modelling the result in one shot. It computes through one event (OneShot) a configuration where all network nodes have unique identities. Formally, this enumeration can be specified as a bijection $(\nrightarrow)$ from the set of nodes $N(G)$ to $\{1,2, \ldots,|N(G)|\}$. If $N D$ is an abstract set specifying $N(G), \operatorname{card}(N D)$ stands for $|N(G)|$. enumeration is an abstract variable introduced for computing the final configuration of the network. It is a function computed on $N D$ which, starting with a uniform identity of nodes, say 0 (see the INITIALIZATION event), eventually terminates with a bijection being an enumeration of nodes (see the OneShot event).

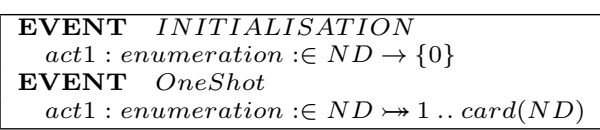

\subsection{The First Refinement}

This level remains in a high level abstraction. It refines the previous one and computes the result in a progressive way. New variables are introduced: enum is a function computed on $N D$, being the enumeration under definition; and maxId is an integer, initialized to 0, encoding the largest value of the current nodes identities. Let $n$ be a node, we assume that enum $(n)$ does not exceed $\max I d$. These variables are specified as follows:

$\operatorname{inv} 1: \max I d \in 0 \ldots \operatorname{card}(N D) \wedge$ enum $\in N D \rightarrow 0 \ldots \max I d$

The idea is to define nodes identities in a progressive way ensuring that all values being in $[0 . . \max I d]$ or in $[1 . . \max I d]$ are currently allocated. For the sake of simplicity, we distinguish two cases:

- the 0-identity still exists in the current configuration. Formally, we ensure that enum $\triangleright\{0\} \neq \varnothing$. Note that enum $\triangleright\{0\}$ is a range restriction that contains all of the pairs that are in enum and whose second element is equal to 0 . In such a case, we ensure the following invariant (inv2): enum is a total surjection $(\rightarrow)$ from $N D$ to $0 . . \max I d$. It is surjective; means that for every element of $0 . . m a x I d$ there exists an element in $N D$ that is mapped to it; and it is total means that its domain contains all elements of $N D$.

inv2: enum $\triangleright\{0\} \neq \varnothing \Rightarrow$ enum $\in N D \rightarrow 0 \ldots \max I d$

- the 0-identity does not exist in the current configuration: enum $\triangleright\{0\}=\varnothing$. In such a case, we ensure the following invariant (inv3): enum is a total surjection from $N D$ to 1..maxId. 
Consequently, we can ensure the following properties:

- the minimum value of the current node identities is either a 0 -identity or a 1-identity (th1).

- if there exists an identity that is mapped to two different nodes equally, then the current configuration of node identities does not contain the value of $\operatorname{card}(N D)$ yet. More precisely, the value of $\max I d$ is strictly less than $\operatorname{card}(N D)(t h 2)$.

$\operatorname{th} 1: \min (\operatorname{ran}($ enum $)) \in\{0,1\}$

th2 : $\forall x, y \cdot\{x, y\} \subseteq N D \wedge x \neq y \wedge \operatorname{enum}(x)=\operatorname{enum}(y) \Rightarrow \max I d<\operatorname{card}(N D)$

A new event enumProgress is introduced to change node identities and update maxId during enumeration. If the current configuration contains a node, say $x$, that is still in its initial state (0-identity) ( $\operatorname{rrd} 2)$, or if all nodes changed their identities (enum $\triangleright\{0\}=\varnothing$ ) but the current configuration assigns a same identity to two different nodes: $x$ and another one, say $y$, $y \in N D \backslash\{x\}(\operatorname{grd} 2)$; then $x$ is mapped to an another identity, say $n$ (act 1$)$. The new value $n$ is selected in such a way that the range of enum increases towards covering all values being in $[1 . . \operatorname{card}(N D)]$. To this end, we ensure that $n \in \operatorname{enum}(x)+1 \ldots \max I d+1(\operatorname{grd} d)$. In such a case, maxId should not obviously exceed $\operatorname{card}(N D)(\operatorname{grd} 3)$. If the new value $n$ exceed the largest value of the current node identities, $\max I d$ sets to the value of $n$. In another words, we ensure that the new value of $\max I d$ takes the largest identity $\max (\{\max I d, n\})(\operatorname{act} 2)$.

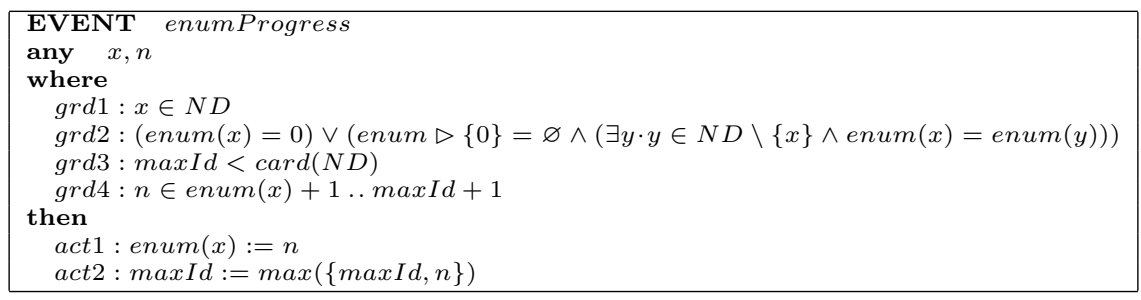

\subsection{The Second Refinement}

For any node $x$ of the graph $g$, we take a local view on the ball around $x$, denoted $B(x)$, and we prove an injection from elements of $B(x)$ to their identities, excepting nodes that are still mapped to 0: two distinct nodes of $B(x)$ are always mapped to distinct identities. For instance, as presented in Fig. 4, $B(x)$, covers the nodes, $x, z, y$ and $t$. The ball around $y$, denoted $B(y)$ covers $y, x, k$ and $w$. In such a case, different inequalities should be ensured between node identities. For example: $\operatorname{enum}(x) \neq \operatorname{enum}(y) ; \operatorname{enum}(x) \neq \operatorname{enum}(k) ; \operatorname{enum}(y) \neq \operatorname{enum}(z)$; $\operatorname{enum}(w) \neq \operatorname{enum}(x)$, etc. We can say that every node should not be mapped to an identity assigned to one of its neighbours being in a distance $\leq 2$. More formally, we model these

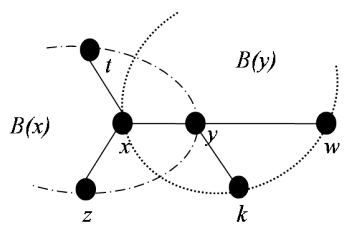

Figure 4: Balls in the graph

requirements with the following invariants and theorems: given $x$ a node of the graph $g, g[x]$ is a subset of $N D$ containing all of neighbours of $x$. Fore every connected nodes of the graph $g$, say $x$ and $y$, such that enum $(x)=$ enum $(y)$, we have to ensure that these nodes did not change their identities yet. They still have the 0-identity (inv4). If there is another node $z$, connected to $x$, such that $z \neq y$ and enum $(z)=\operatorname{enum}(y)$, then $\operatorname{enum}(z)=0($ inv5 $)$. 
$\operatorname{inv} 4: \forall x, y \cdot x \mapsto y \in g \wedge \operatorname{enum}(x)=\operatorname{enum}(y) \Rightarrow \operatorname{enum}(x)=0$

inv5: $\forall x, y, z \cdot z \in g[\{x\}] \wedge y \in g[\{x\}] \wedge z \neq y \wedge \operatorname{enum}(z)=\operatorname{enum}(y) \Rightarrow \operatorname{enum}(z)=0$

Consequently, we can prove that for every other node $w$ connected to $y$, such that $w \neq x$ and $\operatorname{enum}(w)=\operatorname{enum}(x)$, then $\operatorname{enum}(w)=0(t h 3)$. Therefore, we prove that for every node $x$, the function enum computed on the ball around $x$, excepting nodes with 0 -identity, is injective (th4). The domain restriction of enum on $B(x)$ is specified by $(\{x\} \cup g[\{x\}]) \triangleleft$ enum. The range restriction on non 0 -identities is specified by $((\{x\} \cup g[\{x\}]) \triangleleft$ enum $) \triangleright\{0\}$. The theorem th4 ensures that the function enum with its domain/range restriction belongs to a partial injection $(\nrightarrow)$, from $N D$ to $1 \ldots \max I d$. Note that the partial property of this function stems from the fact that the ball around $x$ is a subset of $N D$.

$$
\begin{aligned}
& \text { th3 : } \forall x, y, w \cdot x \in g[\{y\}] \wedge w \in g[\{y\}] \wedge w \neq x \wedge \text { enum }(x)=\operatorname{enum}(w) \Rightarrow \text { enum }(w)=0 \\
& t h 4: \forall x \cdot x \in N D \Rightarrow((\{x\} \cup g[\{x\}]) \triangleleft \text { enum }) \triangleright\{0\} \in N D \succ 1 . \max I d
\end{aligned}
$$

No new variables are introduced in this model. We refine the enumProgress event of the previous machine by two events: enum0 and enum. The first one models the case where a node does not get an identity yet $(\operatorname{grd} 2)$. Guards are strengthened by grd5 to ensure that $x$ will take a different value comparing to its neighbourhood identities. For any node $x$ of the graph $g, g[x]$ is a subset of $N D$ containing all of neighbours of $x ; g[g[x]]$ is the neighbourhood of $x$. It is a subset of $N D$ containing elements of $g[x]$ and their neighbours.

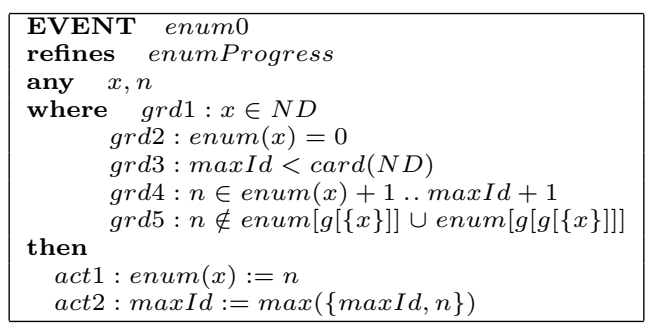

The second event enum models the case where two connected nodes get the same identity (grd2). Similarly to the previous event, guards are strengthened by grd5 to ensure that $x$ will take a different value comparing to its neighbourhood identities.

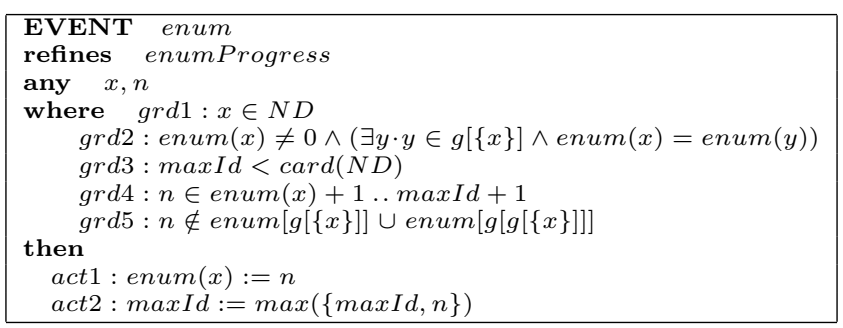

\subsection{The Third and Fourth Refinements}

The third level describes the instructions that every node of $B(x)$ has to follow when $x$ modifies its identity. Events of the previous level, enum 0 and enum, are refined respectively by two other events enum $0^{\prime}$ and enum $^{\prime}$. Each refined event models the updating action of the Lcoal view of $B(x)$ elements. When $x$ changes its number from enum $(x)$ to $n$, then every node $y$, connected to $x$ must update its Local view from $L v(y)$ to $L v^{\prime}(y)$ by removing enum $(x)$ and adding $n$ to $L v(y)$. From all information it can be gathered from neighbour identities and their Local views, every node $x$ of the graph can construct its MailBox, denoted by $M b(x)$. The last level introduces the instructions that a node has to follow to construct its MailBox and make it up to date at every computation step. Events of the previous level, enum $0^{\prime}$ and enum', are refined respectively by 
renaming 0 and remaining. The first event models the first case of the Renaming rule, i.e., when a node has a 0 -identity.

The remaining event refines enum' and models the second case of the Renaming rule, i.e., a node $x$ discovers the existence of another node $y$ mapped to the same identity as $n(x)$. In such a case, $x$ should compare its Local view Lv(x) with the Local view stored in its Mailbox (N0), and decide if it picks another number or not. A new event Diffusion is introduced to compute the Diffusion rule. Finally, we can strengthen the guards of the Oneshot event in order to ensure requirements that yields to a final enumeration: if the $B(x)$ elements have the same Mailbox; and if for every node $x$ of the graph $\operatorname{enum}(x) \neq 0$; and if the identity of $x$ is already stored in its Mailbox and mapped to another Local view, weaker than $n(x)$; then all events are blocked excepting the Oneshot that should yield to a bijection. In order to ensure the correctness of the bijection result, we verify that for every graph say $H$ constructed from $g$ node identities, such that enum is a surjective homomorphism from $g$ to $H$, then $g$ and $H$ are isomorphic via enum. A detailed formal development of our approach is available ${ }^{1}$.

\section{Conclusion and Future Work}

Throughout this paper, we investigated Mazurkiewicz's algorithm, based on local computations, and we proposed an incremental reasoning for managing the complexity of the development and preserving properties between the different abstraction levels. These levels are related by refinement, so that the properties and the behaviour of any model are kept in all its subsequent refinements. This technique supports the step-by-step development and provides an easy way to carry on the correctness of the proofs and to validate the integration of requirements. During development, a set of so-called proof obligations is generated by the Rodin platform. Some of these proofs are discharged automatically by Rodin provers and some need to be discharged interactively.

The computed result constitutes basic initial steps of many other distributed algorithms. Therefore, it can be used, by composition with other algorithms, to give solutions of distributed problems. Combining verified components is one way to overcome the complexity of proving a distributed algorithm. Therefore, numerous future works can be done on this area. As a part of our other efforts, we investigate snapshot problems with multiple initiator using the studied enumeration algorithm, by referring to new theories published in [8]. Finally, we think that investigating enumeration problems with complex distributed systems like mobile networks would be an interesting case study.

\section{References}

[1] Jean-Raymond Abrial. Modeling in Event-B - System and Software Engineering. Cambridge University Press, 2010.

[2] Jean-Raymond Abrial, Michael J. Butler, Stefan Hallerstede, Thai Son Hoang, Farhad Mehta, and Laurent Voisin. Rodin: an open toolset for modelling and reasoning in event-b. STTT, 12(6):447-466, 2010.

[3] Manamiary Bruno Andriamiarina, Dominique Méry, and Neeraj Kumar Singh. Revisiting snapshot algorithms by refinement-based techniques. Comput. Sci. Inf. Syst., 11(1):251-270, 2014.

\footnotetext{
${ }^{1}$ http://visidia.labri.fr/html/formal-development.html
} 
[4] Dana Angluin. Local and global properties in networks of processors (extended abstract). In Proceedings of the 12th Annual ACM Symposium on Theory of Computing, April 28-30, 1980, Los Angeles, California, USA, pages 82-93, 1980.

[5] Ralph Johan Back. A calculus of refinements for program derivations. Acta Inf., 25(6):593-624, 1988.

[6] Paolo Boldi and Sebastiano Vigna. An effective characterization of computability in anonymous networks. In Distributed Computing, 15th International Conference, DISC 2001, Lisbon, Portugal, October 3-5, 2001, Proceedings, pages 33-47, 2001.

[7] Jérémie Chalopin, Emmanuel Godard, and Yves Métivier. Election in partially anonymous networks with arbitrary knowledge in message passing systems. Distributed Computing, 25(4):297$311,2012$.

[8] Jérémie Chalopin, Yves Métivier, and Thomas Morsellino. On snapshots and stable properties detection in anonymous fully distributed systems (extended abstract). In Structural Information and Communication Complexity - 19th International Colloquium, SIROCCO 2012, Reykjavik, Iceland, June 30-July 2, 2012, Revised Selected Papers, pages 207-218, 2012.

[9] Brahim Hamid and Mohamed Mosbah. A local enumeration protocol in spite of corrupted data. JCP, 1(7):9-20, 2006.

[10] Igor Litovsky, Yves Métivier, and Eric. Sopena. Handbook of graph grammars and computing by graph transformation. chapter Graph Relabelling Systems and Distributed Algorithms, pages 1-56. World Scientific Publishing Co., Inc., River Edge, NJ, USA, 1999.

[11] Antoni W. Mazurkiewicz. Distributed enumeration. Inf. Process. Lett., 61(5):233-239, 1997.

[12] Dominique Méry. Refinement-based guidelines for algorithmic systems. Int. J. Software and Informatics, 3(2-3):197-239, 2009.

[13] Dominique Méry and Neeraj Kumar Singh. Analysis of DSR protocol in event-b. In Stabilization, Safety, and Security of Distributed Systems - 13th International Symposium, SSS 2011, Grenoble, France, October 10-12, 2011. Proceedings, pages 401-415, 2011.

[14] G. Tel. Introduction to Distributed Algorithms. Cambridge University Press, 2000.

[15] Mohamed Tounsi, Mohamed Mosbah, and Dominique Méry. Proving distributed algorithms by combining refinement and local computations. ECEASST, 35, 2010. 